\title{
EDITORIAL
}

\section{Soil-Plant Microbiome: a promising frontier for research}

The role of soil microorganisms in nutrient cycles, soil health and plant growth has long been recognized. The traditional microbiologists have focused on a single microbial species and its function at a time. Of them, mycorrhizal fungi, nitrogen fixing bacteria and plant growth promoting rhizobacteria have received relatively more attention due to many applications in agriculture. In recent times, microbiologists have broadened their vistas further by introducing a novel approach with the concept of 'microbiome'. Though the term 'microbiome' was first cited in 1988 (Whipps et al., 1988), the research on 'microbiome' has gathered its momentum much later. Marchesi and Ravel (2015) defined the term 'microbiome' as 'the entire community of microorganisms (bacteria, archaea, lower and higher eukaryotes and viruses) within a habitat and its surrounding environmental conditions'. Due to its complex nature, the scientists endorse a holistic approach to explore how microbiomes influence ecosystems, biosystem functions and their dependence on each other.

The microbes show heightened activities in 'hotspots' in the soil environment such as rhizosphere, mycosphere, drillosphere and/or detritusphere. Out of them, rhizosphere (the region of the soil influenced by plant roots and their exudates) and its inhabiting microorganisms influence the growth of plants positively through production of growth regulatory compounds, thus creating the rhizospheric microbiome a highly evolved external locale for plants. Due to its close link, the rhizospheric microbiome is considered as a second genome to a plant, proposing that a plant is not an individual at its genomic level but a broader genetic entity which comprised of its associated microbial genome (Gopal and Gupta, 2016). This insight has eventually given rise to the concept of 'holobiont', an assemblage of an individual and its symbionts living and functioning as a single unit (Theis et al., 2016). This intimate association between the plant and soil microbiomes is driven mainly by chemical signals produced by the plant (Lebesis et al., 2015), thus providing evidence to suggest that plants shape the structure of rhizospheric microbiome through root exudates.

Complex network interactions of microbes are important in the sustenance of the soil-plant system. It has been reported that generally keystone taxa and members are rich in the rhizosphere soil of healthy plants compared to their diseased counterparts (Huang et al., 2019). However, agricultural intensification reduces network complexity and the abundance of keystone taxa in the root microbiome (Banerjee et al., 2019). This brings challenges to scientists to find ways to sustain high crop productivity under agricultural intensification while saving the microbial networks. Microbial interventions such as biofilm biofertilizers have shown promise, as biofilm action increases rhizosphere soil microbial diversity and abundance while maintaining high crop yields, with reduced chemical inputs (Seneviratne and Kulasooriya, 2013).

The isolation and characterization of microbes using different culture media and growth conditions are no longer considered as effective methods in studying microbial diversity. With the novel concept of microbiome, the scientists have recognized the demand in developing more sophisticated technologies to explore the unseen world of microbes. The discovery of molecular techniques which involved direct extraction and characterization of microbial communities, and metagenomic studies have revolutionized the field of microbial ecology. In particular, metagenomics provides ability to simultaneously characterize complex DNA mixtures from a specific microbial habitat and estimate the metabolic capacity of a given microbial community. The development of high throughput sequencing and new tools for analyzing metagenomic data has helped scientists to tread on areas that are not touched before. As a result, metagenomics have paved the way to access 'un-discovered' genomes in the world of microbes, leading to the discovery of new phyla, classes, genera and genes (Nesme et al., 2016). Metagenomics also help to explore how soil microorganisms alter their populations with changing edaphic properties, interactions and sensitivity to climate change. Such recent advances in high-end technologies have paved the way for a new era in microbiome research providing scientists ample opportunities to explore microbial communities and their functions.

With increasing knowledge on plant-soil microbiomes and their functional capacities, researchers are now diverting their efforts to capitalize on some of the favourable microbial traits to introduce more sustainable next-generation agriculture. However, the lack of organized effort to transfer new findings into practice is largely blamed on the lack of partnership between researchers and farmers, which is even more challenging in less developed countries due to many inherent factors. Despite encouraging results on the use of microbial inoculants as bio-fertilizers (Singhalage et al., 2018), the large-scale applications under field conditions have not yet provided equally promising results, mostly due to unseen interactions with the native flora. However, a recent study has shown encouraging results of using biofilm biofertilizers in rice with $50 \%$ usage of recommended chemical fertilizer (personal communication). This is where the knowledge on plant-soil microbiomes considered vital. 
The next-generation farmers may secure both geochemical and microbiome reports of their farming lands before applying the precise microbial inoculant to optimize crop yields while reducing the usage of costly agrochemicals. Furthermore, the manipulation of soil-plant microbiome has shown promising potential in reducing pests and diseases, promoting fitness and growth of plants. The importance of further research of holistic understanding of plant-soil microbiome to enhance yield and agricultural sustainability has also been emphasized. The use of 'metaproteomics' that allows establishing the genotype-phenotype linkages from in situ samples has many environmental applications as well. Unfortunately, many benefits of soil microbiome are rather susceptible to changing environmental conditions and land management practices. Manipulation of soil microbiome to restore ecosystem functions has also been tested. However, such manipulations can only be possible if we know how soil microbiomes perform under varying conditions. Therefore, it is important to understand the functions of soil microbiome in situ. To address this issue, Jansson and Hofmockel (2018) defined the term 'metaphenome' which is the product of expressed functions encoded in microbial metagenome and the environment. Due to its high complexity together with high spatial and temporal variability, the studies related to 'metaphenome' pose significant challenges to scientists. However, such knowledge will definitely help researchers to develop new approaches to optimize soil nutrient cycles, manage nutrient transport and sustain crop yield under varying conditions.

The wonders of the green revolution in agriculture have reached its pinnacle, leaving scientists with no options to enhance the crop productivity further to meet the everincreasing food demand. This problem has even become more complicated with other on-going issues such as climate change, desertification, depleting soil productivity etc. Therefore, the future of the global agriculture depends on the utilization of microorganisms (Hussain et al., 2018). In spite of promising applications, many knowledge gaps and challenges still exist. The complex nature of microbiomerelated studies and the need of high-end technologies are some of the challenges face by scientists. The use of tractable synthetic communities with modifications to microbial, host and environmental parameters allow scientists to broaden their knowledge and translate this information into applications in agriculture (Vorholt et al., 2017). Finding suitable microbial consortia, delivery techniques and formulations need further investigations before field applications (Compant et al., 2019). The urgency of speeding up studies to find ways to exploit plant-soil microbiome to increase crop productivity and to lessen losses due to biotic and abiotic stresses is imperative in order to meet the future food demand. Though, the understanding of soil-plant microbiome and its potential uses have been vastly increased over the past few years, the scientists still need to find ways to overcome challenges of using microbial inoculants in field conditions, eventually leading to the food security.

H.M.S.P. Madawala

Email: sumedha.madawala@gmail.com

\section{References}

Banerjee, S., Walder, F., Büchi, L., Meyer, M., Held, A.Y., Gattinger, A., Keller, T., Charles, R. and Van Der Heijden, M.G. (2019). Agricultural intensification reduces microbial network complexity and the abundance of keystone taxa in roots. The ISME Journal, https://doi.org/10.1038/s41396-019-0383-2.

Gopal, M. and Gupta, A. (2016). Microbiome selection could spur next-generation plant breeding strategies. Frontiers in Microbiology 7: 1971. doi: 10.3389/fmicb. 2016. 01971.

Huang, X., Zhou, X., Zhang, J. and Cai, Z. (2019). Highly connected taxa located in the microbial network are prevalent in the rhizosphere soil of healthy plant. Biology and Fertility of Soils https://doi.org/10.1007/ s00374-019-01350-1.

Jansson, J.K. and Hofmockel, K.S. (2018). The soil microbiome - from metagenomics to metaphenomics. Current opinion in Microbiology 43: 162-168.

Hussain, S.S., Mehnaz, S. and Siddique, K.H.M. (2018) Harnessing the Plant Microbiome for Improved Abiotic Stress Tolerance. In: Egamberdieva D., Ahmad P. (eds) Plant Microbiome: Stress Response. Microorganisms for Sustainability, vol 5. Springer, Singapore.

Lebeis, S.L., Paredes, S.H., Breakfield, D.S., Gehring, J., McDonald, M. et al. (2015). Salicylic acid modulates colonization of the root microbiome by specific bacterial taxa. Science 349: 860-864.

Marchesi, J. R. and Ravel, J. (2015). The vocabulary of microbiome research: a proposal. Microbiome 3: 31 .

Nesme, J., Achouak, W., Agathos, S.N. et al. (2016). Back to the future of Soil Metagenomics. Frontiers in Microbiology 7: 73. doi: 10.3389/fmicb.2016.00073.

Seneviratne, G. and Kulasooriya, S. A. (2013). Reinstating soil microbial diversity in agroecosystems: The need of the hour for sustainability and health. Agriculture, Ecosystems and Environment 164: 181-182.

Singhalage, I.D., Seneviratne, G., Madawala, H.M.S.P. and Wijepala, P.C. (2019). Profitability of strawberry (Fragaria ananassa) production with biofilmed biofertilizer application. Scientia Horticulturae 243: 411-413.

Theis, K.R., Dheilly, N.M., Klassen, J.I., Brucker, R.M., Baines, J.F., Bosch, T.C. et al. (2016). Getting the hologenome concept right: an eco-evolutionary framework for hosts and their microbiome. mSystems 1:e00028-16. Doi: 10.1101/038596.

Vorholt, J.A., Vogel, C., Carlstrom, C.I. and Muller, D.B. (2017). Establishing causality: Opportunities of synthetic communities for plant microbiome research. Cell Host \& Microbe 22: 142-155.

Whipps, J. M., Lewis, K., and Cooke, R. C. (1988). Mycoparasitism and plant disease control, in Fungi in Biological Control Systems, ed N. M. Burge (Manchester University Press), 161-187. 